\title{
SIGNIFICADOS ALREDEDOR DEL TEJIDO DE PELUCAS ONCOLÓGICAS EN MUJERES DIAGNOSTICADAS CON CÁNCER
}

\author{
MEANINGS BEHIND THE SEWING OF MEDICAL WIGS IN WOMEN DIAGNOSED \\ WITH CANCER
}

\author{
Juan Pablo Sanabria', Andrea Stefanía Muriel' y Nathalia Parra² \\ 1 Pontificia Universidad Javeriana, Cali (Colombia) \\ 2 Funcáncer, Cali (Colombia)
}

Resumen

Fundamento: La pérdida de cabello se constituye como una alteración física que puede influir en la autoestima de mujeres con cáncer.

Objetivo: Comprender los significados alrededor del tejido de pelucas oncológicas en mujeres diagnosticadas con cáncer pertenecientes a un grupo de autoayuda en Cali (Colombia).

Método: Estudio cualitativo de diseño fenomenológico interpretativo en el que participaron 10 mujeres. Los resultados preliminares se presentaron en dos grupos focales conformados por todas las integrantes del grupo, quienes contribuyeron en el análisis de los resultados recolectados previamente en las 10 entrevistas en profundidad. El análisis de los datos se realizó con el apoyo del programa Atlas.Ti.

Resultados: Las participantes describieron la pérdida del cabello como una experiencia traumática de alto impacto emocional. El uso de accesorios les permitió ocultar y reservarse la enfermedad. Las motivaciones para vincularse al grupo se relacionaron con la oportunidad de compartir experiencias con otras pacientes y con el deseo de mejorar tanto su bienestar como el de mujeres con cáncer. Además, pertenecer al grupo les permitió contar con una red de apoyo en la que pudieron expresar sus miedos e inquietudes.

Conclusiones: Aunque la caída del cabello no genera implicaciones funcionales para el organismo, se ha identificado que sobre esta

\section{Abstract}

Fundament: Losing hair is a physical change that could affect the self-esteem of women with cancer.

Objective: Understand the meanings behind the sewing of medical wigs in women diagnosed with cancer belonging to a self-help group in Cali (Colombia).

Methodology: Interpretative phenomenological qualitative study in which 10 women participated. Preliminary results were presented in two focus groups composed of all members of the group who contributed to the analysis of the previously results collected in the $\mathbf{1 0}$ interviews. The data analysis was done with the support of Atlas.Ti program.

Results: Participants described hair loss as a traumatic experience that involved high emotional impact. The use of accessories allowed to hide and reserved the disease. The motivations for linking the group were related to the opportunity to share experiences with other patients and with the desire to improve well-being in themselves and in women with cancer. Also, belonging to the group allowed to have a support network where they could express their fears and concerns.

Conclusions: Although hair loss does not generate functional implications for the organism, it has been identified that this part of the body is related to social representations

\section{Correspondencia:}

Juan Pablo Sanabria Mazo

Funcáncer. Carrera 40 \#5B-64, Cali (Colombia)

Teléfono: 3865560, Ext. 4035 / 4012

E-mail: jsanabria22@javerianacali.edu.co

psicologia@funcancer.com

\section{Información de la investigación:}

Esta investigación fue financiada por Funcáncer y contó con la colaboración de la Pontificia Universidad Javeriana. Los autores del artículo declaran no tener conflictos de intereses derivados de este trabajo. 
parte del cuerpo se inscriben representaciones sociales vinculadas al significado de ser mujer. La actividad de tejido se presenta como un espacio terapéutico en el que las participantes desarrollan estrategias para afrontar aspectos de su vida.

Palabras clave: Cabello, feminidad, cáncer, significados, autoestima, grupos de autoayuda, investigación cualitativa. that includes the meaning of being a woman. The activity of sewing is presented as a therapeutic space where the participants can develop strategies for coping aspects of their life.

Keywords: Hair, femininity, cancer, meanings, self-esteem, self-help groups, qualitative research.

\section{INTRODUCCIÓN}

Los índices de mortalidad y de morbilidad registrados anualmente sitúan al cáncer como uno de los principales problemas de salud pública a nivel mundial. En el año 2012, la Organización Mundial de la Salud reportó 14 millones de nuevos casos y 8.2 millones de defunciones asociadas con la enfermedad ${ }^{(1)}$. En Colombia, según el informe del Plan Decenal para el Control del Cáncer, durante el año 2010 se registraron cerca de 33450 muertes, cifra que representa el $16.9 \%$ del total de defunciones en el país ${ }^{(2)}$.

La literatura resalta que el cáncer genera repercusiones en las dimensiones física, psicológica, social y espiritual de las personas afectadas $^{(3-5)}$. Generalmente, los pacientes perciben este diagnóstico como una experiencia traumática que genera tanto dificultades en los procesos de adaptación como cambios emocionales ${ }^{(7-9)}$. Las alteraciones físicas que producen los tratamientos oncológicos se convierten en factores estresores que las pacientes enfrentan durante la enfermedad $^{(10-12)}$. Los cambios permanentes, como los efectos provocados por las cirugías, y los temporales, como la pérdida de cabello, pueden influir negativamente en la autoestima de las mujeres, generar desórdenes afectivos, problemas en la identidad de género y cambios en su vida sexual ${ }^{(13,14)}$.

En este contexto, la quimioterapia, la radioterapia y las cirugías se presentan como los tratamientos oncológicos que más im- plicaciones tienen en la imagen corporal, pues suponen para las mujeres la pérdida de partes del cuerpo que simbolizan femi$\operatorname{nidad}^{(15,16)}$. Por ejemplo, los medicamentos utilizados en la quimioterapia afectan el crecimiento de las células del organismo y pueden ocasionar efectos secundarios como la alopecia temporal. Como consecuencia de variables relacionadas con el tratamiento, como el medicamento, la dosis y la vulnerabilidad psicológica del paciente, el folículo piloso queda inactivo, lo que ocasiona que las células capilares dejen de proliferar y el cabello caiga temporalmente ${ }^{(15-18)}$.

Recientes investigaciones señalan que los cambios que se producen en las zonas afectadas por los tratamientos tienen implicaciones psicológicas como la sobrevaloración de la importancia estética en el atractivo físico y personal, la insatisfacción corporal, la disminución de bienestar y el miedo al rechazo. Estos efectos pueden generar, en las pacientes, comportamientos de evitación o compensación hacia su cuerpo y su entorno social ${ }^{(6,10,19-22)}$.

Aunque la caída del cabello no genera implicaciones funcionales para el organismo, se ha identificado que sobre esta parte del cuerpo las mujeres inscriben representaciones sociales vinculadas al significado de género ${ }^{(6,10,17)}$, que suelen estar influidas por diferentes variables sociales. Los medios de comunicación, por ejemplo, resaltan en la publicidad la importancia del 
cabello como símbolo de belleza, juventud, lozanía, salud, creencias religiosas, profesión y condición social(23,24). Las prácticas culturales, específicamente en occidente, dan lugar a rituales en los que se resaltan el cabello como un elemento de belleza, de feminidad y de identidad que se debe cuidar para lucirlo en la cotidianidad ${ }^{(17,24-27)}$.

Muchas mujeres que pierden el cabello como consecuencia de un tratamiento oncológico deben continuar con sus rutinas sociales, lo que implica que tengan que enfrentar el estigma social vinculado a la ausencia de cabello ${ }^{(4,19,25,28,30)}$. Las respuestas de la sociedad frente a estos cambios físicos pueden convertirse en barreras que dificultan la aceptación que tienen de su nueva imagen corporal y la forma de interactuar con los otros ${ }^{(19,29,30)}$. Investigaciones en el tema han resaltado que las miradas de rechazo, curiosidad, vergüenza o distanciamiento que reportan las pacientes las impulsan a aislarse de su entorno social y a buscar estrategias para ocultar sus manifestaciones físicas ${ }^{(10,21,23,26-28)}$. Para minimizar este impacto algunas utilizan pelucas, turbantes o gorros con los que pueden ocultar la pérdida de su cabello ${ }^{(21,23,26)}$. El uso de estos elementos les permite evitar que sean identificadas como pacientes con cáncer, lo que las protege tanto a ellas como a sus acompañantes de las manifestaciones no deseadas de su entorno social $^{(25,26,28)}$.

Las alteraciones físicas derivadas de la enfermedad afectan la representación que ellas tienen de su cuerpo e inciden en la vinculación física y afectiva que establecen con los demás ${ }^{(4,9,15,27)}$. En este orden de ideas, se ha planteado que variables como la respuesta y el apoyo del entorno social, el acceso a recursos o procedimientos para amortiguar el impacto de los cambios físicos y la valoración que las pacientes realicen de la alteración física, condiciona la aceptación que tengan las mujeres de su nueva imagen $^{(10,14)}$. El significado que atribuyen a factores sociales y culturales, sumado a sus habilidades de afrontamiento y las características de su personalidad, permiten que tengan una mejor aceptación de su imagen corporal y que consigan ajustarse con mayor facilidad a la enfermedad $3(3,8,14,28)$.

En el año 2014 la fundación Funcáncer creó el programa Banco Solidario de Pelucas como un grupo de autoayuda en el que mujeres diagnosticadas o sobrevivientes de cáncer se reúnen para tejer pelucas oncológicas que van dirigidas a otras mujeres que han perdido el cabello como consecuencia de su tratamiento. En las interacciones que se producen durante la actividad de tejido comparten los significados que ellas atribuyen al proceso de la enfermedad ${ }^{(19,31-33)}$. Uno de los elementos centrales de esta investigación fue la posibilidad de explorar la construcción de los significados que este grupo otorga a las experiencias y las emociones que elabora durante este proceso.

En este sentido, el objetivo de este artículo fue comprender los significados alrededor del tejido de pelucas oncológicas en mujeres diagnosticadas con cáncer pertenecientes al programa Banco Solidario de Pelucas, en Cali (Colombia). Para ello se exploraron los significados asociados al cabello, se identificaron las motivaciones para vincularse al grupo de tejido y se caracterizaron las experiencias relacionadas con esta actividad. Los investigadores de este trabajo entienden que conocer los significados que atribuyen a este tejido es un insumo valioso para comprender el proceso de su enfermedad, reconocer sus estrategias de afrontamiento e identificar su bienestar psicológico.

\section{MÉTODO}

\section{Diseño de estudio}

Esta investigación se realizó desde un enfoque cualitativo de diseño fenomenológico interpretativo, pues este abordaje 
permite describir el significado de las experiencias que perciben las participantes de su realidad y reconoce la función constructiva del investigador en la interpretación de sus experiencias ${ }^{(34)}$.

\section{Participantes}

Participaron 10 mujeres diagnosticadas con cáncer, pertenecientes al programa del Banco Solidario de Pelucas, con edades comprendidas entre los 39 y los 68 años $(M=56.72 ; D E=8.47)$. El tiempo promedio transcurrido desde que se les comunicó el diagnóstico osciló entre dos y 20 años $(M=8.1 ; D E=5.78)$. De esta cantidad, el $60 \%$ de ellas fueron diagnosticadas con cáncer de mama, el $10 \%$ de cérvix, el $10 \%$ de colon, el
$10 \%$ de tejido blando y el $10 \%$ de leucemia mieloide. La totalidad de las participantes recibieron tratamiento durante alguna fase de la enfermedad, siendo la quimioterapia $(70 \%)$, la cirugía $(60 \%)$ y la radioterapia $(50 \%)$ los procedimientos oncológicos más frecuentes. Como consecuencia de dichos tratamientos, el $70 \%$ reportaron haber presentado alopecia en alguna etapa de la enfermedad.

En el momento en que se realizó esta investigación, el $60 \%$ de las participantes se encontraban en fase de remisión y el $40 \%$ aún recibían tratamientos oncológicos (ver tabla 1). La mayoría de las participantes eran solteras $(70 \%)$ y desarrollaban su actividad laboral como amas de casa $(80 \%)$, con un nivel de escolaridad superior $(60 \%)$. En cuanto a su estatus so-

\section{Tabla 1. Características de las participantes}

\begin{tabular}{|l|l|l|l|l|l|}
\hline Pseudónimo & Edad & Diagnóstico & Tratamientos & Fase enfermedad & Alopecia \\
\hline Laura & 65 & Cáncer de colon & $\begin{array}{l}\text { - Quimioterapia } \\
\text { - Radioterapia }\end{array}$ & En remisión & Sí \\
\hline Ana & 50 & Cáncer de mama & $\begin{array}{l}\text { - Quimioterapia } \\
\text { - Radioterapia } \\
\text { - Cirugía }\end{array}$ & En proceso & Sí \\
\hline Paula & 39 & Cáncer de mama & $\begin{array}{l}\text { - Quimioterapia } \\
\text { - Radioterapia } \\
\text { - Cirugía }\end{array}$ & En remisión & Sí \\
\hline Marta & 61 & Cáncer de mama & $\begin{array}{l}\text { - Quimioterapia } \\
\text { - Radioterapia } \\
\text { - Cirugía }\end{array}$ & En proceso & Sí \\
\hline Sofía & 68 & Cáncer de mama & $\begin{array}{l}\text { - Quimioterapia } \\
\text { - Cirugía }\end{array}$ & En proceso & Sí \\
\hline Carmen & 50 & Cáncer de mama & $\begin{array}{l}\text { - Quimioterapia } \\
\text { - Radioterapia } \\
\text { - Cirugía }\end{array}$ & En remisión & Sí \\
\hline Alejandra & 49 & Cáncer tejido blando & $\begin{array}{l}\text { - Quimioterapia } \\
- \text { Radioterapia } \\
- \text { Cirugía }\end{array}$ & En proceso & Sí \\
\hline Isabel & 53 & Leucemia mieloide & - Quimioterapia & En remisión & No \\
\hline María & 66 & Cáncer de cérvix & $\begin{array}{l}\text { - Quimioterapia } \\
- \text { Radioterapia }\end{array}$ & En remisión & No \\
\hline Lucía & 65 & Cáncer de mama & $\begin{array}{l}\text { - Quimioterapia } \\
\text { En remisión }\end{array}$ & No \\
\hline
\end{tabular}


cioeconómico ${ }^{1}$, el $40 \%$ pertenecían al nivel bajo (estrato 1 y 2 ) y el $60 \%$ al medio (estrato 3 y 4$)$.

\section{Técnicas de recolección}

Las técnicas de recolección de información empleadas en este estudio fueron la entrevista en profundidad y los grupos focales. Las guías de preguntas utilizadas contaron con la validación de tres jueces expertos en Psicología de la Salud, quienes verificaron la pertinencia, la coherencia y la redacción de las preguntas, según los objetivos de la investigación y las categorías de análisis. A partir de los comentarios realizados por los jueces y la aplicación de una entrevista piloto, se ajustaron las preguntas que conformaron la guía final de la entrevista (anexo 1).

\section{Procedimiento}

La propuesta de investigación se presentó a las voluntarias que forman parte del Banco Solidario de Pelucas entre noviembre de 2015 y marzo de 2016 y se desarrolló con aquellas que expresaron interés en participar. Una vez firmado el consentimiento informado en el que se les notificó los alcances del estudio, se les entregó una ficha de datos sociodemográficos, con 10 preguntas que incluían datos biográficos, estado civil, nivel de escolaridad, estatus socioeconómico y ocupación. Inicialmente, se contactaron a 10 mujeres que accedieron ser entrevistadas en profundidad. En estas entrevistas se exploraron como categorías de análisis: (1) los significados atribuidos al cabello, (2) las experiencias en el tejido de pelucas oncológicas y (3) las motivaciones para vincularse al programa (ver tabla 2). La elección de las categorías y de las subcategorías de análisis se realizó a partir tanto de las experiencias reportadas por la población de estudio como de los referentes conceptuales identificados en la revisión de la literatura y de los objetivos propuestos en esta investigación.

\section{Tabla 2. Categorías de análisis}

\begin{tabular}{|l|l|}
\hline Categorías & Análisis \\
\hline Significados atribuidos al cabello & $\begin{array}{l}\text { Se tuvieron en cuenta los cambios de los significados en torno } \\
\text { al cabello que se derivaron de experiencias como la pérdida } \\
\text { del mismo y el tejido de pelucas oncológicas }\end{array}$ \\
\hline $\begin{array}{l}\text { Motivaciones para vincularse al } \\
\text { programa }\end{array}$ & $\begin{array}{l}\text { Se exploraron las motivaciones que reportan las participantes } \\
\text { para interesarse, vincularse y mantenerse en el programa de } \\
\text { tejido de pelucas oncológicas }\end{array}$ \\
\hline $\begin{array}{l}\text { Experiencias en el tejido de } \\
\text { pelucas oncológicas }\end{array}$ & $\begin{array}{l}\text { Se investigaron experiencias que se desarrollan en el grupo } \\
\text { de tejido como el proceso de integración, las conversaciones } \\
\text { entre las integrantes, los beneficios asociados al grupo y el } \\
\text { significado atribuido a la actividad }\end{array}$ \\
\hline
\end{tabular}

1 En Colombia el Departamento Administrativo Nacional de Estadística (DANE) realiza una estratificación socioeconómica que se divide en seis rangos. El rango 1 y 2 corresponde al nivel socioeconómico bajo, el 3 y el 4 al medio y el 5 y el 6 al alto. En esta investigación, el estatus socioeconómico fue autoreportado.
El informe preliminar de los resultados obtenidos en las categorías establecidas se presentó en un primer grupo focal conformado por las 10 participantes de las entrevistas en profundidad. Para asegurar la validación de la información interpretada 
Anexo 1. Guía de entrevista

\begin{tabular}{|c|c|}
\hline Categorías & Preguntas \\
\hline \multirow{3}{*}{$\begin{array}{l}\text { Motivaciones } \\
\text { para vincularse al } \\
\text { programa }\end{array}$} & 1. ¿Cómo conoció usted el programa del Banco Solidario de Pelucas (BSP)? \\
\hline & 2. ¿Qué la motivó a vincularse a este grupo de tejido de pelucas? \\
\hline & 3. ¿Qué la motivó a mantenerse en este grupo? \\
\hline \multirow{6}{*}{$\begin{array}{l}\text { Experiencia de } \\
\text { tejido de pelucas }\end{array}$} & 4. ¿Qué ha significado para usted formar parte de este grupo? \\
\hline & 5. ¿Qué siente que le ha aportado el grupo? \\
\hline & 6. ¿Cómo ha sentido que ha sido su proceso de integración al grupo? \\
\hline & 7. ¿Qué sentimientos surgen en usted al realizar esta actividad? \\
\hline & 8. ¿Qué significa para usted tejer una peluca oncológica? \\
\hline & $\begin{array}{l}\text { 9. ¿Considera que el ser una integrante de este grupo le ha ayudado a } \\
\text { afrontar algunos aspectos de su vida? Por favor, cuénteme cómo. }\end{array}$ \\
\hline \multirow{6}{*}{$\begin{array}{l}\text { Significados } \\
\text { alrededor del } \\
\text { cabello }\end{array}$} & 10. ¿Qué importancia considera que tiene el cabello para una mujer? \\
\hline & 11. ¿Qué significado tenía el cabello antes del diagnóstico? ¿Y ahora? \\
\hline & 12. ¿Cómo vivió la experiencia de la caída de su cabello? \\
\hline & $\begin{array}{l}\text { 13. ¿Utilizó algún recurso cuando perdió el cabello? En caso de que su } \\
\text { respuesta sea afirmativa, ¿cuáles? }\end{array}$ \\
\hline & $\begin{array}{l}\text { 14. ¿Cómo cree que los otros perciben la falta de cabello en una mujer } \\
\text { con cáncer? }\end{array}$ \\
\hline & $\begin{array}{l}\text { 15. ¿Cómo han cambiado sus significados alrededor del cabello con el } \\
\text { tejido de pelucas? }\end{array}$ \\
\hline
\end{tabular}

por los investigadores, se solicitó inicialmente a estas participantes que verificaran los resultados obtenidos en las entrevistas individuales, haciéndolas, de esta forma, partícipes en la construcción del análisis. Luego, los investigadores volvieron a analizar la información triangulada con las participantes y en un segundo grupo focal pidieron a 10 tejedoras que, como jueces externas al proceso de recolección de datos, corroboraran los resultados obtenidos. Las instrucciones que se les dieron a las participantes de ambos grupos fue que indicaran si estaban de acuerdo o en desacuerdo con los hallazgos de la investigación. Esta propuesta metodológica permitió, por un lado, triangular en dos momentos diferentes la interpretación que realizaron los investigadores de los resultados con las participantes y las tejedoras y, por el otro, generar un espacio para la devolución de la información obtenida a todo el grupo.

Análisis de datos

Las entrevistas se realizaron en consultorios de la fundación y tuvieron una duración aproximada de hora y media. Estas entrevistas se grabaron en audio y después se transcribieron textualmente. Con el objetivo de validar los resultados de la investigación y reducir posibles sesgos en la interpretación, el análisis de los datos fue realizado, por cada uno de los investigadores, de manera manual basándose en las categorías de análisis y los objetivos de estudio. Así pues, se siguió el esquema de organización, segmentación y correlación, a partir del cual se realizó un primer trabajo de transcripción y codificación con cada una de las piezas, 
seguido de un trabajo con las categorías de análisis y una orientación hacia la construcción del análisis final. Posteriormente, se complementó el análisis de esta información mediante el programa Atlas.Ti (versión 7.5) y se sometió a un proceso de codificación que permitió visualizar los resultados de manera gráfica y por subcategorías.

Los resultados obtenidos se discutieron en un grupo conformado por los tres investigadores y por un juez externo a la investigación, quien se encargó de realizar una retroalimentación de los resultados. De este modo, triangular la información por investigadores evitó que sus sesgos interfirieran en la codificación de la información. La triangulación por investigadores se alcanzó por medio de la técnica Consensual Qualitative Research (CQR), la cual permitió a los investigadores realizar procesos de codificación independientes que se revisaron conjuntamente ${ }^{(35)}$. Esto posibilitó ampliar las miradas y no limitarse a la experiencia de uno de los investigadores, así como facilitar la comparación sistemática y estandarizada en la observación de las influencias de otros investigadores en el problema y los resultados de la investigación.

Esta investigación estuvo orientada por la resolución No. 008430 de 1993 del Ministerio de Salud que expone las normas científicas, técnicas y administrativas para la investigación en salud con seres humanos a nivel nacional. Del mismo modo, se tuvieron en cuenta las consideraciones éticas definidas en el Manual Deontológico y Bioético de Psicología y la Ley 1090 del 2006 que regula el ejercicio de la Psicología en Colombia.

\section{RESULTADOS}

A continuación se presentan los resultados obtenidos, de acuerdo con los objetivos y las categorías de análisis propuestas en esta investigación.
Significados del cabello

Las participantes describieron la pérdida de su cabello como una experiencia traumática que les implicó un alto impacto emocional: "Lloraba de día y de noche. Me decían que no llorara por eso, pero es muy difícil evitarlo" (Ana, 50 años). Para ellas, los aspectos más difíciles de este proceso fueron reconocer esta pérdida como una consecuencia inevitable del tratamiento, sentir la caída progresiva del cabello y experimentar los sentimientos generados por no poder controlar esta situación: "Ver cómo a uno se le cae el cabello por montones... eso es terrible (...) lo más difícil para mí era peinarme y que se me quedara el cabello en las manos. Amanecer y ver en la almohada mi cabello, no sé cómo explicarlo con palabras, pero fue algo traumático" (Laura, 65 años).

Además, manifestaron haber presentado alteraciones en el estado de ánimo al tener que explicar a su círculo social los motivos por los que habían perdido el cabello: "Me sentía desilusionada, triste; cuando mis nietas me preguntaban: 'mamita, ¿por qué no tienes cabello?', era realmente devastador" (Carmen, 50 años). Para otras, en cambio, lo más complicado de la pérdida fue no haberse sentido apoyadas por sus parejas: "Yo en ese tiempo vivía con el papá de mis hijas y me dijo 'no se vaya a hacer esa quimioterapia, ni la radio, porque eso se le va a caer el pelo y va a quedar bien fea, como un hombre. Y yo no quiero verla así, calva'"' (Ana, 50 años).

Algunas manifestaron que la pérdida de cabello fue un tema de conversación que decidieron omitir a nivel familiar por la resistencia que les generaba hablar de esta situación: "Ellos sabían lo difícil que era para mí estar calva y por eso ninguno hacía comentarios al respecto" (Marta, 61 años). Al hablar de la pérdida, los familiares decidieron minimizar el valor que tenía el cabello en su vida y destacaron la 
necesidad de priorizar la importancia de la salud sobre la imagen: "Me decían que no me preocupara por eso, que lo importante era mi salud, que el cabello volvía a crecer tarde o temprano" (Paula, 39 años). Otros, en cambio, las motivaron a emplear estrategias para mitigar el impacto de la pérdida: "Cuando empezó a caérseme [el cabello], mi hermana me dijo: 'Carmen, hágase calvear para que quede el pelo parejito, hágase un corte de acuerdo a lo que está viviendo, así le será menos difícil', y sí, le hice caso" (Carmen, 50 años).

Las participantes que perdieron el cabello en más de una ocasión, expresaron que al iniciar nuevamente el tratamiento eligieron cortarse el cabello y recurrir a accesorios como pelucas, gorros o pañoletas para disminuir las implicaciones emocionales que se derivan de la pérdida: "Para no volver a ver que se me cayera el cabello, fui a donde una peluquera y le dije rápeme toda de una vez, no quiero vivir lo mismo de la primera vez"' (Laura, 65 años). En la segunda experiencia de pérdida, las participantes aseguraron que la relevancia estética que tenía esta parte del cuerpo había disminuido: "Ya no es tan relevante el pelo, hay otras cosas más importantes en mi vida" (Paula, 39 años). Al crecer nuevamente el cabello, identificaron nuevas formas de lucirlo que antes de la enfermedad no habían considerado: "Yo nunca en la vida había tenido el cabeIlo corto, entonces para mí fue muy rico sentirme liberada" (Carmen, 50 años).

Aunque las participantes mencionaron que inicialmente la pérdida de cabello les había generado alteraciones emocionales, ellas decidieron aceptar esta situación como una parte del proceso que debían experimentar si querían recuperarse: "Cuando se me cayó el cabello, yo sentí muchísima nostalgia; no es que no me doliera perder el cabello, claro que me dolió, pero con el tiempo entendí que esto era parte de lo que debía vivir, que no debía ocultarlo, tan solo tenía que vivirlo" (Sofía, 68 años). Ahora bien, ellas aseguraron que de los cambios corporales que surgen como consecuencia de los tratamientos oncológicos, la caída del cabello fue uno de los que les generó mayor impacto en la percepción que tenían de sí mismas: "Los cambios que se viven son muchos y muy intensos, pero la pérdida de cabello fue el que más me afectó... es difícil, uno no se ve nada favorable sin el cabello" (Ana, 50 años).

Como consecuencia de la pérdida del cabello las participantes afirmaron haberse sentido expuestas a los comentarios y las miradas de curiosidad de otras personas: "La gente me empezó a preguntar que por qué me había cortado el cabello (...) Ellos empiezan a interrogarlo a uno, a sentir lástima... y eso me frustraba mucho" (Marta, 61 años). En este sentido, expresaron que la ausencia del cabello en una mujer genera reacciones que reflejan la percepción del cáncer como una enfermedad contagiosa asociada directamente con la muerte: "Cuando lo ven a uno sin cabello, lo primero que dicen es 'ay, esa persona tiene cáncer', se va a morir..." (Laura, 65 años). Ante esto, reconocieron que preferían no frecuentar espacios públicos para evitar comportamientos o comentarios que las hiciera sentir incómodas: "Uno siente que las personas te miran raro, que se alejan porque piensan que las van a contagiar (...) todo esto lo entristece a uno $y$, al final, uno le coge miedo a salir y prefiere encerrarse en su casa" (Carmen, 50 años).

Las participantes resaltaron que el cabello es un parte del cuerpo que cumple un papel importante en la percepción que tienen de su estado de salud, de su autoestima y del reconocimiento social: "Tener cabello es muy importante para uno, para nuestra autoestima; es lo que nos permite como mujeres vernos bien frente a los demás. Por eso hay que llevarlo bien arreglado, bien bonito, para no verse desvali- 
do, no verse enfermo" (Laura, 65 años). El cabello les permitía mostrarse como mujeres y sentirse satisfechas consigo mismas: "El cabello y los senos son las etiquetas que tenemos las mujeres, nuestra carta de presentación (...) Sin cabello uno puede llegar a verse como si fuera un hombre" (Ana, 50 años).

Para ellas, el cabello fue un símbolo de belleza que les posibilitaba reconocerse como mujeres, diferenciarse del sexo opuesto, adquirir estatus social y mostrarse como personas saludables: "El cabello es lo que nos permite a nosotras como mujeres diferenciarnos de los demás, es algo muy nuestro, muy propio (...) Si una tiene el cabello bien arreglado o bien puestecito tiene buena admiración, lo miran a uno y piensan esta señora está bien" (Carmen, 50 años). Alrededor del cabello las participantes generaron prácticas de cuidado que denotan la importancia que tiene esta parte del cuerpo para ellas: "Mantenía mi cabello bien cuidado, compraba el mejor champú, me gustaba alisarlo, mantenerlo siempre bien bonito (...) A mí me encantaba que me acariciaran el cabello y es algo que hacía mi mamá conmigo y que hago yo ahora con mi hijos" (Marta, 61 años).

Como una medida para ocultar la ausencia del cabello, hicieron uso de pelucas, pañoletas o gorros. La mayoría de ellas afirmaron que tomar la decisión de utilizar uno $u$ otro accesorio estuvo influenciado por la posibilidad económica que disponían en ese momento. Algunas que contaron con estos recursos aseguraron preferir usar únicamente gorros y pañoletas al considerar el uso de las pelucas como un insumo artificial: "Aprendí a colocarme las pañoletas y los gorritos (...) mi familia me preguntó que si quería una peluca sintética, pero yo les dije que no, que no me gustan las cosas que no son naturales" (Ana, 50 años). Otras decidieron utilizar estos accesorios porque les permitía diferentes combinaciones para vestir, a la vez que les posibilitaba camuflar la enfermedad que estaban viviendo: "Ahora venden pañoletas y gorros de todos los colores. ¿Tú qué haces?, tratas de conjuntar tu gorrito dependiendo de la ropa que tengas (...) Mucha gente pensaba que yo usaba los gorros de pura vanidad, y yo me reía y les decía que sí, que era exactamente por eso" (Carmen, 50 años). En otros casos, manifestaron evitar el uso de gorros o pañoletas porque estos accesorios son socialmente asociados con la enfermedad o porque les recuerda experiencias previas relacionadas con su proceso: "Las personas ven a alguien con un gorro o una pañoleta y directamente piensan que uno tiene cáncer. Por eso yo decidí utilizar únicamente pelucas" (Alejandra, 49 años).

La mayoría afirmaron que las pelucas se utilizan como una estrategia para ocultar el proceso de enfermedad y reservar esta información solamente para personas cercanas: "Con las pelucas una puede disimular que tiene cáncer. Yo lo hice porque quería evitar la curiosidad de la gente, no quería que la gente supiera; quería sentir que podía decidir a quién decirle y a quién no lo que tenía" (Laura, 65 años). Otras mencionaron que por momentos el uso de las pelucas les permitió olvidarse de la enfermedad, sentirse mejor consigo mismas y evitar la lástima de los demás: "Yo con mi peluquita sentía como si no me estuviera pasando nada, salía con normalidad, dejaba de ver la lástima en la cara de la gente; a veces hasta me olvidaba de lo que estaba pasando" (Alejandra, 49 años). Como consecuencia de la enfermedad las participantes aseguraron experimentar el estigma social relacionado con el cáncer.

\section{Motivaciones para vincularse al grupo}

Las participantes afirmaron conocer el programa Banco Solidario de Pelucas por recomendación de otras personas, referencia de profesionales de salud de otras 
instituciones, convocatoria interna, vinculación de otros programas que ofrece la fundación y publicidad en medios de comunicación. Las motivaciones para pertenecer al grupo se relacionaron con el deseo que tenían de aprender una nueva actividad, con el interés de formarse en la actividad de tejido de pelucas y con la oportunidad de ayudar a otras personas: "Yo quería ubicarme en un grupo donde yo pudiera hacer algo, donde yo pudiera servirle a personas que lo necesitaran" (Isabel, 53 años). Otras, en cambio, se vincularon al grupo por el deseo de compartir con mujeres que han pasado por el mismo proceso: "Me gustó el hecho de que uno en este espacio pueda escuchar la experiencia de otras compañeras que han vivido lo que yo viví" (Lucía, 65 años).

El principal motivo para vincularse a esta actividad fue la oportunidad de brindar pelucas oncológicas a mujeres que perdieron el cabello como consecuencia del cáncer. Las dificultades que muchas tuvieron para adquirir una peluca durante su tratamiento por no contar con los recursos económicos necesarios fue lo que las motivó a interesarse por esta actividad: "No tuve dinero para comprar una peluca. Eso es lo que más me motivó a ayudar a estas mujeres, que si no tienen la posibilidad de comprarla puedan pedirla. Porque, a decir verdad, una peluca de pelo natural como la que nosotros tejemos, cuesta mucho dinero" (Sofía, 68 años).

Entre las principales razones para continuar en el programa se encontró el deseo de generar bienestar a mujeres con diagnóstico de cáncer y la oportunidad de compartir experiencias con pacientes que están pasando por la enfermedad: "Uno con pequeñas cosas le aporta al mundo. El beneficio más que para sí mismo es para la gente que se beneficia del programa" (María, 66 años). El apoyo recibido por parte de las demás voluntarias y los vínculos de amistad que se generaron fue lo que las impulsó a continuar asistiendo al grupo: "Lo bien que la paso yo acá, para mí es motivo de mucho disfrute, yo gozo, me río mucho" (Sofía, 68 años).

\section{Experiencias en el tejido de pelucas}

Durante el aprendizaje del tejido algunas participantes refirieron haber tenido dificultades iniciales debido a la falta de práctica en la manipulación del cabello, no obstante fueron las tejedoras con más experiencia quienes actuaron como facilitadoras en la enseñanza de esta actividad: "Las nuevas llegan y las que llevamos más tiempo nos acercamos a ellas para enseñarles lo que deben hacer: colocar los hilos, armar el pelo, ajustar las pelucas... las que llegan aportan algo que no sabíamos a las que estábamos aquí y una les aporta a ellas cosas que no sabían" (Lucía, 65 años). En muchos casos aprender a tejer cabello fue considerado por las integrantes del grupo como un logro personal que les generaba satisfacción y reconocimiento de sus habilidades en el trabajo manual: "Yo siempre he sido torpe para las manualidades (...) nunca en la vida había hecho algo como esto, entonces sentir que estaba transformado el cabello en algo tan bonito, eso me hizo sentir como la persona más importante del mundo" (Carmen, 50 años). Una de ellas manifestó que esta actividad le había ayudado a recuperar la funcionalidad motriz que había perdido como consecuencia de su enfermedad: "A mí me ha servido mucho porque yo tengo un pequeño temblor en las manos. Obviamente tengo mis días difíciles, días en los que me tiemblan más las manos que otras veces; sin embargo, haciendo las pelucas yo me siento bien, lo puedo hacer y por eso yo digo que esto a mí me ha ayudado mucho" (Sofía, 68 años).

La interacción que se produjo entre las integrantes del grupo les permitió modificar creencias que tenían en relación con 
la enfermedad: "Al compartir con otras personas una se va dando cuenta que tiene que tener una actitud diferente ante la enfermedad; eso lo va como fortaleciendo, lo va ayudando. Ya no me quejo, he aprendido a vivir este proceso de una forma diferente" (Laura, 65 años). Del mismo modo, pertenecer al grupo les ayudó a contar con una red de apoyo en la que ellas pueden expresar libremente las inquietudes y los miedos que en ocasiones prefieren reservarse: "Aquí puedes hablar, sentir que no te van a juzgar, que no te van a decir $\mathrm{CO}^{-}$ sas que no quieres escuchar, que como hemos vivido lo mismo nos entendemos mejor que si hablamos con otra persona que no haya pasado por esto" (Alejandra, 49 años). Así, las tejedoras que pasaron por el proceso de la enfermedad se convirtieron en un modelo de referencia para aquellas que lo estaban viviendo: "Ver a las compañeras que ya están mejor, más recuperadas, me hace sentir... más esperanzada" (Ana, 50 años).

Otro beneficio que identificaron fue encontrar un espacio exclusivo en el que podían salir de su cotidianidad y volver a sentirse productivas: "Venir aquí es una oportunidad para salir de mi casa, de mi rutina (...) gracias a esta experiencia me he vuelto a sentir útil. El pensar que uno todavía sirve en esta sociedad ha sido bastante gratificante para mí" (María, 66 años). Además, formar parte del grupo les dio la posibilidad de vincularse a otras instituciones en las que podían recibir información u orientación sobre la enfermedad, asistir a los servicios que ofrece la fundación y acceder a citas médicas con especialistas por medio del programa de donación de la institución.

Los temas de conversación que se establecieron durante la actividad del tejido se centraron en aspectos de la cotidianidad y del pasado: "(...) hablamos de nuestra día a día, de nuestros recuerdos, de aspectos que son tan difíciles, pero que hoy en día tomamos con humor" (Sofía, 68 años). Las participantes afirmaron que durante este espacio la enfermedad pasó a un segundo plano y que únicamente conversaron sobre ella cuando alguna de las integrantes requirió algún consejo por parte del grupo: "En realidad no hablamos de lo que nos dio, porque eso es parte del pasado y ya para qué hablar de la enfermedad. Lo que pasa es que nos interesamos por las compañeras que están en el proceso; si hablamos del tema es por ellas, para darles consejos" (Laura, 65 años). Al hablar de la enfermedad, se identificó que sus discursos estaban permeados por el positivismo y el empoderamiento para afrontar el proceso: "Sentimos que estamos bien, que estamos sanas, que Dios nos dio otra oportunidad de cambiar, que podemos salir adelante" (Marta, 61 años).

Las participantes refirieron que el tejido de cabello se convirtió en un espacio que les permitía dejar a un lado sus preocupaciones: "Uno se distrae, esto es una terapia muy grande, porque la casa absorbe, la casa cansa, entonces uno viene y se distrae, se desconecta" (Isabel, 53 años). Esta actividad fue considerada por ellas como una terapia ocupacional que les ofrecía tanto beneficios emocionales como físicos: "Para mí el hecho de tejer y de hacer esa labor me desestresa, uno acá llega y se relaja; eso es un beneficio para mi salud (...) antes creía que la terapia era lo que uno hacía con las manos, pero no, en realidad, es lo que uno hace con la cabeza" (María, 66 años). Con el tiempo la actividad de tejido se convirtió en un espacio reservado en el que podían salir de su cotidianidad mientras compartían con sus compañeras: "El martes no lo toco para nada, siempre y cuando no tenga citas, aquí vengo todas las semanas (...) formar parte de este grupo se ha vuelto en una prioridad para mí" (Paula, 39 años). Ser reconocidas como integrantes del grupo de tejido generó orgullo y sentido de pertenencia por el trabajo realizado: "Me siento 
orgullosa de mí misma (...) cuando yo le cuento a la gente que yo hago esto, todo el mundo me felicita, me dice: ‘iqué labor tan linda!'. Eso me hace sentir realmente bien, me hace sacar pecho, sentirme orgullosa" (Sofía, 68 años).

Algunas participantes manifestaron que por medio del tejido de pelucas reconocieron la importancia del cabello como un elemento valioso que brinda bienestar a las mujeres con cáncer: "Ahora lo valoro más, siento que cada cabello que la gente nos regala hay que cuidarlo, hay que evitar desperdiciarlo (...) se valora más porque es una parte preciosa, una materia prima de felicidad" (Laura, 65 años). Otras mencionaron que situarse en el lugar de las mujeres que habían perdido el cabello les permitió superar la resistencia que tenían a trabajar con esta parte del cuerpo: "Yo no resistía el cabello de las personas (...) cuando empecé le pedí ayuda a mi Dios, no sabía cómo iba a hacer para coger el cabello de otras persona y le cuento que empecé a trabajar divinamente con él, me di cuenta de la importancia que tiene para nosotras como mujeres y así fue como le perdí la fobia" (María, 66 años).

En la entrega de pelucas las tejedoras interactuaron directamente con las pacientes y observaron en ellas el cambio emocional que les producía volver a tener cabello: "Es muy gratificante cada vez que entregamos las pelucas y vemos las caras de felicidad de las señoras. Uno ve el contraste entre la tristeza con la que llegan y la felicidad con la que se van después de ponerles las pelucas (...) Soy feliz al sentir que le levanto la autoestima a mujeres que lo necesitan, que estamos haciendo que se sientan lindas, que no se rindan, que sepan que no por el hecho de que se les vaya a caer el pelo por la quimio dejan de verse bonitas. Que adquirir las pelucas les levante el ánimo, que sea un motivo para seguir viviendo y seguir luchando, un motivo para salir adelante" (Sofía, 68 años).

\section{DISCUSIÓN}

El objetivo principal de esta investigación fue comprender los significados alrededor del tejido de pelucas oncológicas en mujeres diagnosticadas con cáncer que pertenecen a un grupo de autoayuda. En esta medida, el presente trabajo exploró los significados atribuidos al cabello, las motivaciones para vincularse al grupo de tejido y las experiencias relacionadas con esta actividad. Los sistemas de significación permitieron comprender el sentido que este grupo de mujeres construyen en relación con sus historias de vida, sus interacciones y la experiencia de la enfer$\operatorname{medad}^{(36)}$.

Al respecto, se encontró que para las participantes el cabello adquiere representaciones que están asociadas con su identidad de género, apariencia física, su autoestima, estatus social y salud. Como se menciona en la literatura, los movimientos culturales y religiosos históricamente han asignado significados y funciones sobre el cabello ${ }^{(17,27,37)}$. La extensión, el color y el tipo del cabello es un indicador simbólico a través del cual las mujeres y los hombres han inscrito representaciones sociales vinculadas al género, la identidad cultural, el poder, la condición social, la salud y la capacidad reproductiva ${ }^{(24,37)}$. Concretamente, alrededor de esta parte del cuerpo se identifican prácticas culturales de cuidado que destacan el valor que tiene como símbolo representativo de feminidad y de

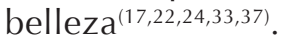

En concordancia con investigaciones anteriores que han explorado los efectos psicológicos generados por los cambios físicos, se encontró que las participantes que perdieron el cabello como consecuencia de los tratamientos describieron esta situación como una experiencia traumática de alto costo emocional ${ }^{(6,10,18-22)}$. Al igual que en los estudios mencionados, se en- 
contró que cambios temporales como la pérdida del cabello afecta en las mujeres la percepción que tienen de su apariencia física, produce insatisfacción con su imagen corporal, implica alteraciones emocionales y genera miedo frente al estigma social $^{(7,8,9)}$. En algunos casos la alopecia derivada de los tratamientos fue percibida por algunas participantes como un recordatorio constante sobre la gravedad de la enfermedad y la presencia de la misma en su vida diaria ${ }^{(22,26,27,28,29,31)}$. También, se identificó que las participantes desarrollaron comportamientos de evitación como no hablar sobre la pérdida del cabello, evadir mirarse al espejo y aislarse socialmente para evitar las reacciones negativas de los otros. El uso de accesorios oncológicos como turbantes, gorros o pelucas se presentó como una estrategia de afrontamiento que permite ocultar la pérdida del cabello, olvidar la enfermedad y controlar que los otros conozcan la presencia del cáncer ${ }^{(21,23,24,28,29)}$.

Como se evidenció en los relatos, el estigma social vinculado a la ausencia del cabello en las mujeres interfirió en la aceptación que ellas tuvieron frente a esta pérdida. Generalmente, las participantes reconocen que la alopecia en la mujer es socialmente asociada con una manifestación del cáncer. La pérdida del cabello, por tanto, deja expuesto ante los demás aspectos de su vida personal, como la enfermedad, que muchas de ellas prefieren conservar para sí mismas ${ }^{(22,24,26,27,28,30)}$. De acuerdo con el estudio de Fontibón et al. ${ }^{(19)}$, se identificó que las expresiones verbales y no verbales de rechazo actuaron como barreras que dificultaron la aceptación que tienen las mujeres de los cambios físicos que deben enfrentar durante la enfermedad.

La participación en el programa Banco Solidario de Pelucas les permitió disminuir los impactos negativos que genera esta pérdida y facilitó la aceptación tanto de su imagen corporal como de la enfermedad. Como se ha reportado en otras investigaciones, la forma de asumir estos cambios físicos estuvo relacionada, en gran medida, con factores individuales como las construcciones, los significados personales, familiares y sociales y las experiencias de pérdidas previas ${ }^{(9,10,22,28,29)}$.

$\mathrm{Al}$ igual que en otras intervenciones realizadas con grupos de autoayuda, los resultados de este estudio evidencian que una de las principales motivaciones de las participantes para asistir al grupo de tejido se relaciona con la búsqueda de apoyo emocional y la posibilidad de ofrecer ayuda a los demás. Compartir experiencias con otras mujeres que han pasado por el cáncer facilita en ellas la expresión de sentimientos y pensamientos, a la vez que les permite resignificar aspectos relacionados con la enfermedad. El contexto grupal posibilita que las participantes se adapten a la enfermedad, desarrollen estrategias de afrontamiento y se ofrezcan apoyo mutuo en aspectos de su vida. La experiencia de las mujeres que han vivido la enfermedad facilita el ajuste de aquellas que lo están viviendo $^{(38,39)}$, a la vez que consolida la autoestima de quienes ya han superado la fase de pérdida de cabello.

Durante la actividad de tejido se producen cambios de actitudes, sentimientos o creencias que facilitan a las participantes aproximarse desde otra perspectiva a las experiencias que han vivido en el transcurso de su enfermedad ${ }^{(38,39)}$. sus discursos durante la actividad de tejido dan cuenta de la reelaboración de procesos internos para afrontar la enfermedad. La participación de las mujeres en el Banco Solidario de Pelucas permite ayudar desde su trabajo manual y experiencias de vida a otras que lo necesitan.

Si bien al principio el cabello cobra un papel de gran importancia en la imagen que tienen las mujeres de sí mismas, en el marco de la actividad de tejido las 
participantes disminuyen la importancia de la función estética que tiene esta parte del cuerpo para priorizar el cuidado de su salud. A partir de la interacción grupal, el cabello pasa de ser un indicador simbólico de feminidad a ser un elemento que, por el impacto que tiene en la autoestima, ofrece bienestar a otras mujeres ${ }^{(38,39)}$. En esta medida, las ceremonias de entrega se configuran como un ritual en el que las tejedoras facilitan pelucas oncológicas a mujeres con cáncer como un elemento que permite amortiguar las implicaciones de la pérdida del cabello tanto para las pacientes como para quienes las acompañan en el proceso de pérdida ${ }^{(24,27,28,29)}$.

En este orden de ideas, el Banco Solidario de Pelucas se constituye como una red de apoyo para las participantes en la medida que les representa beneficios: (a) a nivel instrumental, pues por su participación obtienen recursos o convenios para acceder a tratamientos o atención con especialistas dentro de la misma fundación; (b) a nivel informativo, porque las participantes adquieren conocimientos para enfrentar la enfermedad por medio de la asistencia a capacitaciones, eventos y programas de la fundación; y (c) a nivel emocional, puesto que los vínculos que se establecen entre las integrantes les permite afrontar situaciones personales. Estos recursos posibilitan que las participantes resignifiquen la enfermedad y la experiencia de pérdida del cabello, así como el descubrimiento de recursos internos para afrontar dificultades a nivel personal, familiar y social.

Además, las participantes identifican como beneficios de pertenecer al programa salir de la cotidianidad, sentirse productivas, dejar de lado pensamientos relacionados con la enfermedad y generar impacto social. El tejido se presenta como un espacio terapéutico que les permite expresar, experimentar y validar libremente sus sentimientos, a la vez que mejorar algunas limitaciones motrices derivadas de los tratamientos médicos ${ }^{(38,39)}$. Como se ha planteado en la literatura, los grupos de autoayuda promueven apoyo emocional, ofrecen herramientas para la resolución de problemas y posibilitan retroalimentación a las participantes respecto a la enferme$\operatorname{dad}^{(28-30,38,39)}$.

Una de las principales fortalezas de esta investigación fue la participación activa que tuvieron las tejedoras en el proceso de construcción y validación de los resultados. Esto permitió a los investigadores triangular la información para reflejar la percepción de sus propias experiencias vividas alrededor del tejido de pelucas. De igual manera, se reconoce la inclusión de todas las integrantes que constituyen el grupo como una estrategia que permitió abordar los significados de la actividad del tejido desde las perspectivas de todos sus integrantes.

Teniendo en cuenta que la pérdida de cabello es uno de los cambios físicos que más impacto emocional genera a las mujeres con cáncer, se recomienda que futuras investigaciones continúen explorando los significados que los pacientes construyen alrededor del cabello en el contexto de su enfermedad y, a su vez, evaluar cómo esta pérdida puede influir en la adherencia al tratamiento oncológico. Del mismo modo, se sugiere la implementación de grupos de autoayuda como una estrategia terapéutica para fortalecer los recursos de los pacientes y contribuir al proceso de adaptación de la enfermedad.

El programa Banco Solidario de Pelucas se presenta como un espacio terapéutico en el que las participantes pueden resignificar procesos internos mientras contribuyen al proceso de otras mujeres que están experimentando la enfermedad. Esta red de apoyo posibilita la construcción de vínculos entre diferentes actores sociales (donantes de cabello, tejedoras y fundación) para contribuir al bienestar de pacientes oncológicos. 


\section{AGRADECIMIENTOS}

Los investigadores de este artículo agradecen al grupo de tejedoras del Banco Solidario de Pelucas por permitirles conocer sus experiencias, sentimientos e historias de vida. Del mismo modo, se resalta los aportes de los profesores Diego Emiro Correa y Nathalie Olano durante el desarrollo de esta investigación.

\section{REFERENCIAS}

1. Organización Mundial de la Salud [Internet]. Organización Mundial de la Salud (OMS); 2015 [actualizado feb 2015; citado 3 dic 2015]. Disponible en: http://www. who.int/mediacentre/factsheets/fs297/es/

2. Ministerio de Salud y Protección Social [Internet]. Bogotá: Ministerio de Salud y Protección Social (MPS); 2012 [actualizado marz 2012; citado 3 dic 2015]. Disponible en: http://www.iccp-portal.org/sites/ default/files/plans/PlanDecenal_ControlCancer_2012-2021.pdf

3. Carneiro F, Von Humboldt S, Leal I. Bemestar subjetivo em idosas com e sem cancro da mama em remissão. Rev Psicol Saúde Doen 2014;15:192-201. Doi: 10.15309/14psd150116

4. Emmanuelly $P$, Ferreira, SM. Vivências e práticas de cuidado de mulheres em processo de tratamento de câncer. Rev Ciên Saúde Colet 2015;20:2211-20. Doi: 10.1590/1413-81232015207.18022014

5. Moreno A, Krikorian A, Palacio C. Malestar emocional, ansiedad y depresión en pacientes oncológicos colombianos y su relación con la competencia percibida. Av Psicol Latinonot 2015;33:517-29. Doi: 10.12804/apl33.03.2015.10

6. Cerezo MV, Ortiz M, Cardenal V. Expresión de emociones y bienestar en un grupo de mujeres con cáncer de mama: una intervención psicológica. Rev Latinoam Psicol 2009;41:131-40. Doi: 10.14349/ rlp.v41i1.561
7. Ochoa C, Sumalla EC, Maté J, Castejón V, Rodríguez A, Blanco I, Gil F. Psicoterapia positiva grupal en cáncer. Hacia una atención psicosocial integral del superviviente de cáncer. Psicooncología 2010;7:7-34.

8. Ottati F, Souza MP. Qualidade de vida e estratégias de enfrentamento de pacientes em tratamento oncológico. Act Colom Psicol 2014;17:103-10. Doi: 10.14718/ ACP.2014.17.2.11

9. Schneider J, Pizzinato A, Calderón M. Mujeres con cáncer de mama: apoyo social y autocuidado percibido. Rev Psicol 2015;33: 439-67.

10. Fernández Al. Alteraciones psicológicas asociadas a los cambios en la apariencia física en pacientes oncológicos. Psicooncología 2004;1(2-3):169-80.

11. Neipp M, López S, Terol M, Pastor M. Changes in control beliefs, emotional status and psychosocial adaptation among women with breast cancer. Anal Psicol 2009;25:36-43.

12. Tojal C, Costa R. Ajustamento mental ao cancro da mama: papel da depressão e suporte social. Rev Psicol Saúd e Doen 2014;15(3). Doi: 10.15309/14psd150317.

13. Ortiz E, Méndez L, Camargo J, Chavarro S, Toro G, Vernaza M. Relación entre las estrategias de afrontamiento, ansiedad, depresión y autoestima, en un grupo de adultos con diagnóstico de cáncer. Psychol Avan Discip 2014;8:77-83.

14. Rincón ME, Pérez MÁ, Borda M, Rodríguez AM. Impacto de la reconstrucción mamaria sobre la autoestima y la imagen corporal en pacientes con cáncer de mama. Univer Psychol 2012;11:25-41.

15. Díaz M, Rodríguez M, Trujillo N, Palacios $X$. Cumplimiento con la quimioterapia intravenosa y algunos factores asociados a este en pacientes oncológicos adultos. Avan Psicol Latinonot 2013;31:467-82. Doi: 10.1111/j.15244741.2009.00883.x.

16. Palacios X, Zani B. Representaciones sociales del cáncer y de la quimioterapia 
en pacientes oncológicos. Diver Perspec Psicol 2014;10:207-23. Doi: 10.12804/ apl33.03.2015.09

17. Díaz VE. Sexualidad, cuerpo y duelo: experiencia clínica con mujeres diagnosticadas con cáncer ginecológico o de mama. Pensam Psicol 2010;7:155-60.

18. Posligua AL, Vega, MT, Santos K. Alteraciones del pelo inducidas por quimioterapia. Dermatol Rev Mex 2013;57:382-88.

19. Fontibón L, Acosta L, Valdelamar J, Valdelamar A, Sánchez R. Asociación entre variables sociodemográficas, clínicas y calidad de vida con el apoyo social percibido en pacientes colombianos con cáncer. Av Psicol Latinonot 2015;33:38795. Doi: 10.12804/apl33.03.2015.02

20. Fuentes S, Blasco, T. Factores predictores de la adaptación a la enfermedad en pacientes recién diagnosticadas de cáncer de mama que acuden a un servicio de psicooncología. Anal Psicol 2012;28:73642. Doi: 10.6018/analesps.28.3.156031

21. Parra C, García L, Insuasty J. Experiencias de vida en mujeres con cáncer de mama en quimioterapia. Rev Colomb Psiquiat 2011;40:65-84.

22. Lemieux J, Maunsell E, Provencher L. Chemotherapy induced alopecia and effects on quality of life among women with breast cancer: a literature review. Psychooncology 2008;17:317-28. Doi: 10.1002/pon. 1245

23. Scorsolini F, Santos MA, Souza LV. Vivências e discursos de mulheres mastectomizadas: negociações e desafios do câncer de mama. Estud Psicol 2009;14:41-50. Doi: 10.1590/S1413-294X2009000100006

24. Cash T. The psychology of hair loss and its implications for patient care. Clin Dermatol 2001;19:161-66.

25. Jayde V, Boughton M, Blomfield P. The experience of chemotherapy-induced alopecia for Australian women with ovarian cancer. Eur J Cancer Care 2013;22:503-12.

26. Rosman S. Cancer and stigma: experience of patients with chemotherapy- induced alopecia. Patient Educ Couns 2004;52:333-39.

27. McCorkle R, Ercolano E, Lazenby, M, Schulman D, Schilling LS, Lorig K, et al. Self-management: enabling and empowering patients living with cancer as a chronic illness. CA Cancer J Clin 2011;61:5062. Doi: 10.3322/caac.20093

28. Choi EK, Kim IR, Chang O, Kang D, Nam SJ, Lee JE, et al. Impact of chemotherapy induced alopecia distress on body image, psychosocial well-being, and depression in breast cancer patients. Psychooncology 2014;23:1103-10. Doi: 10.1002/ pon.3531

29. Frith H, Harcourt D, Fussell A. Anticipating an altered appeareance: women undergoing chemotherapy treatment for breast cancer. Eur J Oncol Nurs 2007; 11:385-91.

30. Zannini L, Verderame F, Cucchiara G, et al. 'My wig has been my journey's companion': perceived effects of an aesthetic care programme for Italian women suffering from chemotherapy induced alopecia. Eur J Cancer Care 2012;21:650-60.

31. Palacios X, Lizarazo A, Moreno K, Ospino J. El significado de la vida y de la muerte para mujeres con cáncer de mama. Av Psicol Latinonot 2015;33:455-79. Doi: 10.12804/apl33.03.2015.07

32. Mera P, Ortiz M. La relación del optimismo y las estrategias de afrontamiento con la calidad de vida de mujeres con cáncer de mama. Rev Ter Psicol 2012;30,69-78. Doi: 10.4067/S0718-48082012000300007

33. Galindo Ó, Rojas E, Aguilar JL, Alvarado S, Benjet C, Meneses A. Efectos de intervenciones psicológicas en supervivientes de cáncer: una revisión. Psicooncología 2014;11:233-41. Doi: 10.5209/ rev_PSIC.2014.v11.n2-3.47385

34. Willig C. Introducing qualitative research in Psychology. Berkshire: McGrow Hill House, 2013

35. Hill C, Knox S, Thompson B, Nutt E, Hess S, Ladany $\mathrm{N}$. Consensual qualitative research: 
an update. J Couns Psychol 2005;52. Doi: 10.1037/0022-0167.52.2196

36. Hernández Á. Vínculos, individuación y ecología humana: hacia una psicología clínica compleja. Bogotá, Colombia: Universidad Santo Tomás, 2010.

37. Goicotxea Á. El pelo en la cultura y en la antropología. País Vasco, España: Ediciones Pasto, 2008.
38. Durá E, Hernández S. Los grupos de autoayuda como apoyo social en el cáncer de mama. Las asociaciones de mujeres mastectomizadas de España. Bol Psicol 2003;78:21-39.

39. Suriá SM, Beléndez M. Grupos de apoyo virtuales dedicados a problemas de salud: estudio de su tipología y análisis de representatividad. Anal Psicol 2011;27:210-20. 
